\title{
Serum CA 125, Progesterone, and BHCG Levels in the Differential Diagnosis of Abortion and Ectopic Pregnancy
}

\author{
Sibel MUTLU' ${ }^{1}$, Ali R. BENLi ${ }^{2}$, Orhan GELIŞEN³ ${ }^{3}$ Burcu AYKAN ${ }^{4}$
}

${ }^{1}$ Department of Gynecology and Obstetrics, Hayat Medical Centre, Karabuk, Turkey.

${ }^{2}$ Department of Family Medicine, Karabuk University, Medical Faculty, Karabuk, Turkey.

${ }^{3}$ Department of Gynecology and Obstetrics, Kecioren Medical Centre, Ankara, Turkey.

${ }^{4}$ Obstetrics and Gynecology Clinic, Antalya Educational and Research Hospital, Antalya, Turkey.

\section{ABSTRACT}

This study aimed to investigate the role of serum cancer antigen (CA) 125, progesterone, and beta-human chorionic gonadotropin ( $\beta H C G$ ) levels in the differential diagnosis of intrauterine pregnancy, complete or incomplete abortion, and extrauterine pregnancy.

This study was carried out in a teaching and research hospital between 2007 and 2009. A total of 175 patients with abortion, tubal ectopic pregnancy, and healthy pregnancy were recruited into the study. Pre- and post-treatment blood levels were compared among the groups.

Serum $\beta H C G$ levels were higher in cases of normal healthy pregnancy than in cases of abortion and ectopic pregnancy. No difference was observed between serum BHCG levels of abortion and ectopic pregnancy cases. The levels of serum CA 125 in abortions were found to be higher compared with the other two groups. The levels of serum progesterone in cases of ectopic pregnancy were found to be lower compared with the other two groups.

The utilization of the serum $\beta H C G$ level in the differential diagnosis of normal/abnormal pregnancies and serum CA 125 and progesterone levels in the differential diagnosis of abortion and ectopic pregnancies are alternative parameters.

Key Words: CA 125, complete/incomplete abortion, ectopic pregnancy, $\beta H C G$, progesterone

\section{INTRODUCTION}

In early pregnancy, it is very difficult to distinguish the clinical and laboratory values of normal pregnancies, complete or incomplete abortions, and ectopic pregnancies (1). Late diagnoses can cause troubles that may result in maternal deaths (2). Ectopic pregnancies are still responsible for 10\% of maternal deaths in the Western world and are the leading cause of maternal mortality in the first trimester (3). Therefore, they should be diagnosed as soon as possible so treatment can begin.

The solution to this problem is limited to the interpretation of ultrasonography and blood values. While an ultrasonography is dependent on factors such as the experience of the technician and the quality of the device, unlike subjective parameters, blood parameters can increase correct diagnosis rates (4).

The estimation of gestational wellness using one or more hormonal parameters in early pregnancy has been the subject of many studies about the discrimination of normal and abnormal pregnancies. It has been observed that these blood parameters show differences between these groups of patients. The levels of beta-human chorionic gonadotropin ( $\beta H C G$ ), cancer antigen (CA) 125, and progesterone in these patients are controversial in the literature (5). The aim of this study was to determine the role of serum $\beta H C G, C A 125$, and progesterone levels in the differential diagnosis between abortion and ectopic pregnancies. 
TABLE 1: Demographic characteristics of groups.

\begin{tabular}{|c|c|c|c|c|}
\hline & $\begin{array}{l}\text { Control group } \\
\qquad(n=50)\end{array}$ & $\begin{array}{l}\text { Abortus group } \\
\qquad(n=50)\end{array}$ & $\begin{array}{l}\text { Ectopic pregnancy } \\
\qquad(n=75)\end{array}$ & $p$ \\
\hline Age (year) & $23.6 \pm 3.7(17-34)$ & $26.5 \pm 5.6(18-41)$ & $30.5 \pm 5.2(21-43)$ & $<0.001$ \\
\hline Gravidity & $1(1-4)$ & $2(1-8)$ & $3(1-7)$ & $<0.001$ \\
\hline Mean pregnancy week & $7.1(5-10.4)$ & $7.3(5-12)$ & $5.4(4-9)$ & $<0.001$ \\
\hline
\end{tabular}

\section{MATERIALS AND METHODS}

This study was carried out in a teaching and research hospital between 2007 and 2009, and it was approved by the Institutional Review Board. A total of 175 patients suffering from pelvic pain or vaginal bleeding, with complete/incomplete abortion ( $n=$ $50)$, tubal ectopic pregnancy ( $n=75)$, and healthy intrauterine pregnancy $(n=50)$ were included in the study. Serum $\beta H C G, C A$ 125 , and progesterone levels were compared between these three groups.

The patients were chosen among the intrauterine pregnancies that were proved by ultrasound and resulted in a complete or incomplete abortion less than 12 weeks and tubal ectopic pregnancies that were proved by ultrasound. The control group consisted of patients having an intrauterine pregnancy with a fetal heart rate below 12 weeks. Demographic characteristics, gynecological and obstetric history, and ultrasonographic findings were recorded in the patient evaluation form following the informed consent of the patients. All patients in the study group were assessed by vaginal and ultrasonographic examinations, whereas the patients in the control group were evaluated only by ultrasonography.

Ruptured ectopic pregnancies, pregnancies after infertility treatment, pregnancies with other pathologic conditions that would increase the CA125 level, and pregnancies with progesterone treatment due to abortus imminence were excluded. Since serum BHCG levels could be affected by the gestational week, BHCG levels were corrected according to the gestational week.

At the time of admission, $10 \mathrm{~mL}$ of peripheral blood samples were taken from the antecubital vein for BHCG, CA125, and progesterone levels. Repeat samples were taken for $\beta \mathrm{HCG}$, CA125, and progesterone measurement on days 4 and 7 of single-dose methotrexate therapy for ectopic pregnancies as well as $24 \mathrm{~h}$ after revision curettage that resulted in abortion. The samples were assessed on the same day without waiting. Serum measurements of the three parameters were performed by the radioimmunoassay method with a Rosh E 170 Modular Instrument at the nuclear medicine laboratory of the hospital.

\section{Statistical Analyses}

Data were analyzed using SPSS Software for Windows version 11.5 (SPSS, IL, USA). The Shapiro-Wilk test was used to determine whether the distribution of continuous variables was close to normal. Descriptive statistics were expressed as mean \pm standard deviation for age and as median (minimummaximum) for gravida, gestational week, CA125, progesterone, and BHCG. In the case of a large number of parameters, the receiver operator characteristics (ROC) curve was used to assess the diagnostic importance of each.

\section{RESULTS}

The demographic characteristics of the patients, such as age, gravidity, and gestational week, are given in Table 1. A significant difference was found between the groups in terms of age $(P<0.001)$. The mean age of the ectopic pregnancy group was significantly higher than that of the control and abortion groups $(P<0.001$ and $P<0.001)$. Moreover, the mean age of the abortion group was also higher compared with the control group ( $P=0.013)$. Mean gravida was different between the groups $(P<0.001)$. The gravidity of the abortion and ectopic pregnancy groups was higher than that of the control group ( $P=0.012$ and $P<0.001$ ). No difference was observed between the abortion and ectopic pregnancy groups in terms of mean gravida ( $P=0.073$ ). A significant difference was found between the groups in terms of gestational week $(P<0.001)$. The gestational week of the ectopic pregnancy group was lower than that of the control and abortion groups $(P<0.001$ and $P<0.001)$. No difference was noted between the control and abortion groups in terms of mean gestational week $(P=0.952)$.

A significant difference was observed in terms of CA 125 levels $(P<0.001)$. CA 125 levels were significantly higher in the abortus group than in the control and ectopic pregnancy groups $(P<0.001$ and $P<0.001)$. No significant difference was found between the control and ectopic pregnancy groups in terms of CA 125 levels (Table 2). 
TABLE 2: Comparison of CA125, progesterone and ßhcg levels between groups.

\begin{tabular}{lcccc}
\hline & Control & Abortion & Ectopic pregnancy \\
& Median (min-max) & Median (min-max) & Median (min-max) \\
\hline CA 125 & $27.2(6.5-64.2)$ & $53.6(18.6-743)$ & $21.1(3.2-223)$ & $<0.001$ \\
Progesterone & $23.1(8.7-46.5)$ & $12.6(3.1-31.4)$ & $3.8(0.6-12.3)$ & $<0.001$ \\
Bhcg & $25958.8(2223-182642)$ & $852.5(26-33077)$ & $1400(178-12169)$ & $<0.001$ \\
\hline
\end{tabular}

A significant difference was observed between the groups in terms of progesterone levels $(P<0.001)$. The progesterone levels of the ectopic pregnancy group were significantly lower than those of the control and abortion groups $(P<0.001$ and $P<0.001)$. At the same time, the progesterone level of the abortion group was also significantly lower than that of the control group $(P<0.001)$.

Significant differences were also found between the groups in terms of $\beta H C G$ levels $(P<0.001)$. $\beta H C G$ levels of the abortion and ectopic pregnancy groups were lower than those of the control group $(P<0.001$ and $P<0.001)$. No significant difference was observed between the ectopic pregnancy and abortion groups in terms of BHCG level ( $P=0.144$ ). Because BHCG levels were clinically affected by the gestational week, $\beta H C G$ levels of the ectopic pregnancy and abortus groups remained lower compared with the control group when the effect of the gestational week was ruled out $(P<0.001$ and $P<0.001)$.

It was seen that CA125 was a significant determinant when distinguishing the abortion group from the control and ectopic pregnancy groups. The area under the curve of CA125 (95\% confidence interval) was $0.892(0.840-0.944)$. The best cut-off point was 39.77 . The sensitivity, selectivity, and positive and negative predictive values for this point were, respectively, $86 \%$, $87.2 \%, 72.9 \%$, and $94 \%$ (Figure 1).

When corrected according to the gestational week, the sensitivity, selectivity, and positive and negative predicted values at the cutoff points determined for the BHCG level were not changed while distinguishing the ectopic pregnancy and abortion groups from the control group.

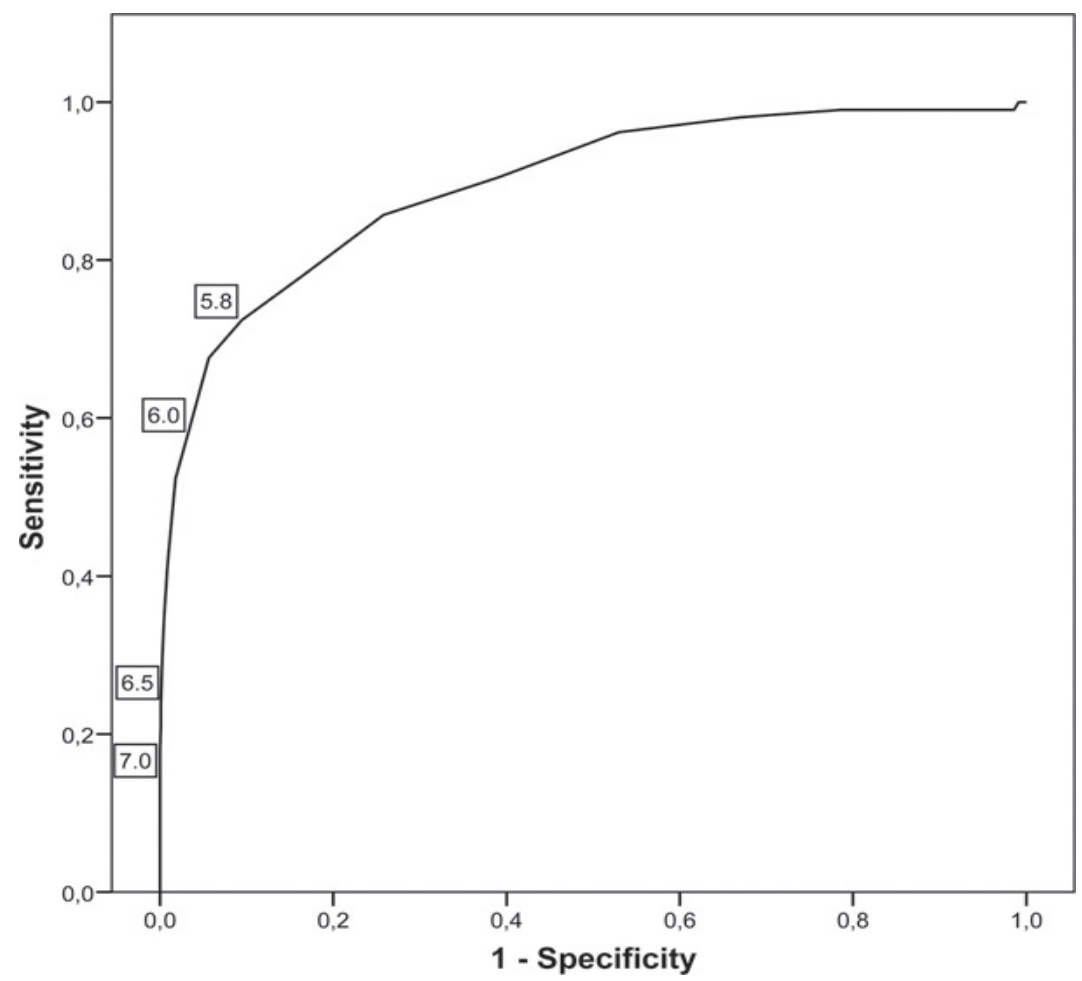

FIGURE 1: ROC curve of serum CA 125 level with ectopic pregnancies. 
A significant decrease was found in CA125, progesterone, and $\beta H C G$ levels after revision curettage within the abortion group, according to the admission $(P<0.001, P<0.001$, and $P<0.001$, respectively).

In the ectopic pregnancy group, after single-dose methotrexate treatment, a statistically significant difference was observed in recurrent $C A 125$, progesterone, and $\beta H C G$ levels $(P<0.001$, $P<0.001$, and $P<0.001)$. A statistically significant decrease was detected in $\mathrm{CA125}$, progesterone, and BHCG levels on the fourth and seventh days compared with the levels on admission $(P<0.001)$. A statistically significant decrease was detected in CA125, progesterone, and BHCG levels on the seventh day compared with the levels on the fourth day $(P<0.001)$.

\section{DISCUSSION}

Abdominal pain and other gynecologic causes of vaginal bleeding should be considered in the differential diagnosis of ectopic pregnancies, one of which is an intrauterine abortion. An anamnesis, physical examination, and ultrasonography are often inadequate in distinguishing these two clinical situations, and some biochemical and hormonal parameters such as serial measurements of $\beta \mathrm{HCG}$ level can be used for a differential diagnosis (6).

Despite variations according to age and gravida, no difference was found between the groups. It is assumed that the situation did not affect the results of the study because it is known that no age-related changes in the examined parameters exist among the patients appropriate for pregnancy.

The gestational week was found to be higher during the first evaluation in the control group because abortion and ectopic pregnancies were detected more frequently in the early weeks of gestation, and the pregnancies were normal in the control group. The BHCG was corrected to fit the gestation week of patients with abortion and ectopic weeks.

The serum BHCG levels of the abortion and ectopic pregnancy groups were significantly lower than those of the control group in this study $(P<0.001, P<0.001)$. No significant difference was observed between the ectopic and abortion groups ( $P=0.144)$.

$\beta H C G$ is a valuable biochemical marker in pregnancy. It is known that first-trimester serial BHCG measurements may be a good parameter to distinguish whether the trophoblastic function isnormal (7). Lower levels than those in the gestational week are important for the diagnosis of ectopic pregnancy (8). Serial BHCG measurements are reported to be more valuable than one $B H C G$ measurement, despite publications that advocate a reversal (9).
Below-normal BHCG measurements in the first trimester predict a spontaneous abortion (10).

Statistically significant differences were observed between groups in terms of CA125 in this study $(P<0.001)$. CA 125 levels of the abortion group were statistically higher than those of the control and ectopic pregnancy groups $(P<0.001, P<0.001)$. No statistically significant difference was found between the control and ectopic pregnancy groups in terms of CA125 levels $(P=0.05)$.

The source of serum CA125 levels in pregnant women is the endometrial glands in the decidua (8). Maternal serum CA125 levels may vary during pregnancy. They increase markedly in the first trimester and then decrease to a normal level in the second and third trimesters (11). Studies have shown that events causing damage to decidua and fetal membranes lead to an increase in maternal serum CA 125 levels (12). Maternal serum CA125 levels may guide determining the prognosis of pregnancy, as well as the degree of damage of the decidual and fetal membranes (13). This describes the increase in CA125 due to the damage in fetal membranes and endometrium in abortions. The literature showed that CA 125 increased in abortions according to normal pregnancies and the relative increase in CA 125 levels because of the lack of fetal membrane and endometrial destruction in normal pregnancy and extrauterine pregnancies until rupture (11). A similar result was found in the present study. On the contrary, other researchers found no difference between abortions and ectopic pregnancies and discerned low levels of CA 125 in relation to normal pregnancies (14). In another study, the CA 125 level was highest in abortions, less in normal, and least in ectopic pregnancies (15). It is thought that this divergence between the studies is due to the difference in the number of pregnancies, the volume of the pregnancy, and damaged tissue volume.

The other parameter in the present study, progesterone hormone, is secreted from the corpus luteum until the seventh week of pregnancy. The placenta starts secreting progesterone with the corpus luteum between the seventh and tenth weeks and continues producing it alone after the tenth week. Low progesterone levels are usually associated with abnormal pregnancies (abortive, ectopic) (16). However, the specific level determining abnormal pregnancy is not known. Serum progesterone levels in ectopic pregnancies are below $15 \mathrm{ng} / \mathrm{mL}$, and this level does not coincide with the levels in normal pregnancies (17). The level in the present study was found to be similar to the mean level of $3.8 \mathrm{ng} / \mathrm{mL}$, and values less than $5 \mathrm{ng} / \mathrm{mL}$ showed nonviability. Serum progesterone levels were below $15 \mathrm{ng} / \mathrm{mL}$ in $81 \%$ of ectopic pregnancies, while serum progesterone levels were below $11 \mathrm{ng} / \mathrm{mL}$ in $11 \%$ 
of normal pregnancies (1). Similar results were obtained in the present study. If the cut-off value is taken as $25 \mathrm{ng} / \mathrm{mL}, 95 \%$ of the cases above this level showed that the pregnancy was normal (18). The present study results were found to be similar to those described in the literature. Some studies showed that it was normal in abortion (19), and some showed that the progesterone level had no prognostic significance (20). It seems that different studies in the literature provide divergent results about the importance of progesterone. Also, the serum $B H C G, C A 125$, and progesterone levels were re-evaluated on the fourth and seventh days of singledose methotrexate treatment in ectopic pregnancies and at the 24th hour after revision curettage in abortion cases. A statistically significant decrease in post-treatment values was observed according to the values at the time of admission $(P<0,001$, $P<0,001)$. Similar results were reported in the literature $(21)$.

Therefore, it is thought that these parameters have a prognostic value in the evaluation of the response to treatment.

Consequently, the present study investigated the prognostic significance of serum $\beta H C G, C A 125$, and progesterone levels in differentiating ectopic pregnancies from intrauterine abortions. Serum $\beta H C G$ levels have prognostic significance in discriminating between normal and abnormal pregnancies. However, it is not an effective and reliable parameter to distinguish abnormal pregnancies. The serum CA125 level can be used as an alternative parameter in discriminating ectopic pregnancies and abortions because it is significantly higher in abortion cases compared with ectopic pregnancy and normal pregnancies. The serum progesterone level has a prognostic value in discriminating ectopic pregnancies and abortions; however, further research is needed on cut-off values.

\section{REFERENCES}

1. Marion LL, Meeks GR. Ectopic pregnancy: history, incidence, epidemiology, and risk factors. Clinical obstetrics and gynecology 2012;55(2):376-86.

2. Mayor S. Early diagnosis of ectopic pregnancy is essential to reduce deaths, says guideline. British Medical Journal 2016:355:i5954.

3. Das R, Biswas S, Roy U. Ectopic pregnancy, Salpingectomy, Maternal mortality. Recurrent ipsilateral ectopic pregnancy following complete salpingectomy 2013;2(32):6011-4.

4. Ayim F, Tapp S, Guha S, Ameye L, Al-Memar M, Sayasneh A, et al. Can risk factors, clinical history and symptoms be used to predict risk of ectopic pregnancy in women attending an early pregnancy assessment unit? Ultrasound in Obstetrics \& Gynecology 2016;48(5):656-62.

5. Eskicioğlu F, Özdemir A, Turan G, Gür E, Kasap E, Genç M. The efficacy of complete blood count parameters in the diagnosis of tubal ectopic pregnancy. Ginekol Pol 2014;85(11):823-7.
6. Casikar I, Reid S, Condous G. Ectopic pregnancy: Ultrasound diagnosis in modern management. Clinical obstetrics and gynecology 2012;55(2):402-9.

7. Korevaar TI, Steegers EA, de Rijke YB, SchalekampTimmermans $\mathrm{S}$, Visser WE, Hofman A, et al. Reference ranges and determinants of total hCG levels during pregnancy: the Generation R Study. European journal of epidemiology 2015:30(9):1057-66.

8. Mavrelos D, Memtsa M, Helmy $S$, Derdelis G, Jauniaux E, Jurkovic D. $\beta$-hCG resolution times during expectant management of tubal ectopic pregnancies. BMC women's health 2015;15(1):1.

9. Daily CA, Laurent SL, Nunley WC. The prognostic value of serum progesterone and quantitative $\beta$-human chorionic gonadotropin in early human pregnancy. American journal of obstetrics and gynecology 1994;171(2):380-4.

10. Barash JH, Buchanan EM, Hillson C. Diagnosis and management of ectopic pregnancy. Am Fam Physician 2014;90(1):34-40.

11. Abdul-Hussein MM, Abdul-Rasheed OF, Al-Moayed HAQ. The values of CA-125, progesterone, ss-HCG and estradiol in the early prediction of ectopic pregnancy. Oman medical journal 2012;27(2):124.

12. Check JH, Nowroozi K, Winkel CA, Johnson T, Seefried L. Serum CA 125 levels in early pregnancy and subsequent spontaneous abortion. Obstetrics \& Gynecology 1990;75(5):742-4.

13. Kobayashi F, Sagawa N, Nakamura K, Nonogaki M, Ban C, Fujii $S$, et al. Mechanism and clinical significance of elevated CA 125 levels in the sera of pregnant women. American journal of obstetrics and gynecology 1989;160(3):563-6.

14. Brumsted J, Nakajima S, Badger G, Riddick D, Gibson M. Serum concentration of CA-125 during the first trimester of normal and abnormal pregnancies. The Journal of reproductive medicine 1990;35(5):499-502.

15. Witt BR, Wolf GC, Wainwright CJ, Johnston PD, Thorneycroft IH. Relaxin, CA-125, progesterone, estradiol, Schwangerschaft protein, and human chorionic gonadotropin as predictors of outcome in threatened and nonthreatened pregnancies. Fertility and sterility 1990;53(6):1029-36.

16. Di Renzo GC, Mattei A, Gojnic M, Gerli S. Progesterone and pregnancy. Current Opinion in Obstetrics and Gynecology 2005;17(6):598-600.

17. Lu $Q$, Li $Y$, Shi $H$, Lang $X$, Wang $Y$. The value of ratio of hCG, progesterone in local blood of pregnancy location versus venous blood in the diagnosis of ectopic pregnancy. International journal of clinical and experimental medicine 2015;8(6):9477.

18. Stovall TG, Ling FW, Cope BJ, Buster JE. Preventing ruptured ectopic pregnancy with a single serum progesterone. American journal of obstetrics and gynecology 1989;160(6):1425-31.

19. Doubilet $P M$, Benson $C B$, Bourne $T$, Blaivas $M$. Diagnostic criteria for nonviable pregnancy early in the first trimester. New England Journal of Medicine 2013;369(15):1443-51.

20. Kant RH, Ara S, Lone AI, Gupta S. Evaluation of outcome of pregnancy in threatened abortion by serum progesterone levels. International Journal of Reproduction, Contraception, Obstetrics and Gynecology 2015;4(5):1313-8.

21. Abdul-Hussein MM, Abdul-Rasheed OF, Al-Moayed HAQ. Receiver operator characteristics and diagnostic value of $C A$ 125 , progesterone, estradiol and $\beta-\mathrm{hCG}$ in the prediction of ectopic and abortive intrauterine gestations Al-Kufa Journal for Biology 2013;5(1):1-6. 